\title{
FY 97 Report on Hydrogen Sensors for Enhanced Surveillance Program Project LL-ESP96-13
}

by

S. E. Nave

Westinghouse Savannah River Company

Savannah River Site

Aiken, South Carolina 29808

This paper was prepared in connection with work done under the above contract number with the U.S. Department of Energy. By acceptance of this paper, the publisher and/or recipient acknowledges the U. S. Government's right to retain a nonexclusive, royalty-free license in and to any copyright covering this paper, along with the right to reproduce and to authorize others to reproduce all or part of the copyrighted paper. 


\section{DISCLAIMER}

This report was prepared as an account of work sponsored by an agency of the United States Government. Neither the United States Government nor any agency thereof, nor any of their employees, makes any warranty, express or implied, or assumes any legal liability or responsibility for the accuracy, completeness, or usefulness of any information, apparatus, product, or process disclosed, or represents that its use would not infringe privately owned rights. Reference herein to any specific commercial product, process, or service by trade name, trademark, manufacturer, or otherwise does not necessarily constitute or imply its endorsement, recommendation, or favoring by the United States Government or any agency thereof. The views and opinions of authors expressed herein do not necessarily state or reflect those of the United States Government or any agency thereof.

This report has been reproduced directly from the best available copy.

Available to DOE and DOE contractors from the Office of Scientific and Technical Information, P. O. Box 62, Oak Ridge, TN 37831; prices available from (423) 576-8401.

Available to the public from the National Technical Information Service, U. S. Department of Commerce, 5285 Port Royal Road, Springfield, VA 22161. 


\section{DISCLAIMER}

Portions of this document may be illegible electronic image products. Images are produced from the best available original document. 
WSRC-RP-97-00904

Rev 0

Keywords: fiber

optics, spectrometers, spectroscopy,

hydrogen, reflectivity

FY97 Report on Hydrogen Sensors for Enhanced Surveillance Program Project LL-ESP96-13 (U)

Stanley E. Nave

Publication Date: September 30, 1997

Westinghouse Savannah River Company Savannah River Site

Aiken, SC 29808

Authorized Derivative Classifier 
CONTENTS

ABSTRACT .......................................................................................................................................1

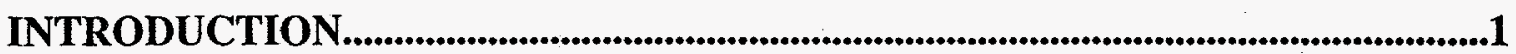

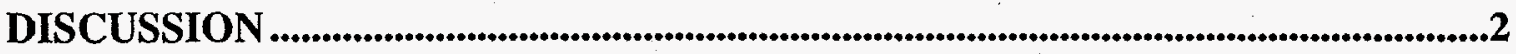

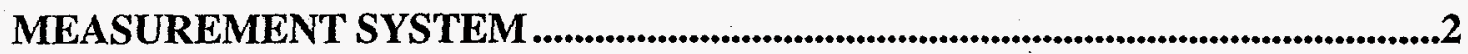

SENSOR COATINGS ...........................................................................................2

SPUTTER SYSTEM..........................................................................................................................2

PURE PALLADIUM AND PALLADIUM ALLOY COATINGS ............................3

YTTRIUM-PALLADIUM LAYERED STRUCTURES ...........................................3

IMPROVEMENTS IN MULTIPLEXING SENSORS...............................................5

CONCLUSION ..................................................................................................................5

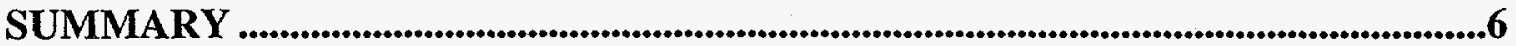


Figure 1. Schematic of the complete fiber optic thin film hydrogen sensor system. .7

Figure 2. Schematic of the thin film palladium sensor. The inset shows a close-up of the layered coatings on the end of the lens(the integral $\mathrm{ZnSe}$ temperature sensor is proposed but is not included for this work)

Figure 3. Reflectivity(counts) of a pure palladium sensor at 635 nanometers versus time(seconds) in response to the indicated pulse concentrations of hydrogen in air

Figure 4. Reflectivity(counts) of a pure palladium sensor at 635 nanometers versus time(seconds) in response to the indicated pulse concentrations of hydrogen in nitrogen. 10

Figure 5. Reflectivity(counts) of a 95\%palladium / 5\% silver sensor at 635 nanometers versus time(seconds) in response to a pulse of .5\% hydrogen in air after setting in air for an extended period. The curve with the initial increase in reflectivity is the uncoated sensor while the curve for which this spike is suppressed is coated with $\sim 1500$ angstroms of teflon.

Figure 6. Reflectivity(counts) of a pure palladium sensor at 635 nanometers versus time(seconds) in response to the indicated pulse concentrations of hydrogen in nitrogen.12

Figure 7. Response of 3000 Angstrom Y/200 Angstrom Pd film to successive hydrogen pulses. $.996 \% \mathrm{H} 2$ in $\mathrm{N} 2$ was used as a sample gas for first run, diluted 1:1 in N2 for second run, and diluted 1:1 in air for the final run. Both peaks are tracked as the sample is let into and released from the chamber, and the red curve below is the amplitude difference between the peak maxima.

Figure 8. Singular Value Decomposition of the response of the Y/Pd film to sequential hydrogen pulses. (a) Score one and two look very similar to the single wavelength magnitude trend and score 3 resembles the difference between the peaks' response profiles. These can be correlated to the wavelength response by examining the corresponding loading vectors (b). The transmission peaks are correlated with each other as hydrogen is added and removed from the cell, but to a differing degree, i.e. in score one the peak at 810 has a higher loading, or a greater response, and for score 2 the peak at $484 \mathrm{~nm}$ has a higher loading. This indicates that they respond at different rates to the presence of hydrogen. The intermediate area, where rapid changes in hydrogen partial pressure occur, is described by loading three, where the two transmission peaks are anticorrelated in their response. This is due to the more rapid response of the peak at $810 \mathrm{~nm}$ relative to that of the peak at $484 \mathrm{~nm}$. This clearly indicates that two mechanisms are present in the optical response of the multilayer Y/Pd hydrogen sensor. 
WSRC-RP-97-00904

\title{
FY97 Report on Hydrogen Sensors for Enhanced Surveillance Program Project LL-ESP96-13 (U)
}

\author{
S. E. Nave and B. B. Anderson \\ Westinghouse Savannah River Company \\ Savannah River Site \\ Aiken, SC 29808
}

\begin{abstract}
A prototype for an all fiber optic hydrogen sensor system was developed. Capability to measure concentrations of hydrogen in air or nitrogen in the range of $0.5 \%$ to $4.0 \%$ with a resolution of $0.1 \%$ was demonstrated. A DC planar magnetron sputter system was procured and assembled for use in the thin metal film deposition necessary for fabrication of the palladium and palladium-silver alloy sensors used in this development. A method was developed to coat the metal films with an organic coating permeable to hydrogen but not oxygen and other reactive gases. The results of tests on these sensors gave positive results but long term studies are required to confirm protection under conditions expected to be encountered in a real world application. A new type of sensor based on a layered yttrium/palladium structure was constructed and tested. The greater magnitude and wavelength dependence of the spectral response observed may lead to a more robust sensor. However, the need to have oxygen present for this sensor in order for it to rapidly recover after exposure to hydrogen may complicate its use.
\end{abstract}

\section{INTRODUCTION}

As part of the Chemically Amplified Optical Sensor ESP initiative with Lawrence Livermore National Laboratory, a robust fiber optic hydrogen sensor system is being developed at SRTC. The major customer requirements include long-term monitoring with a non-electrical sensor element and fiber-optic technology can meet these needs. This system consists of a tungsten-halogen light source and spectrometer to measure the reflectivity of a palladium alloy film to determine hydrogen concentrations in the $0-4 \%$ range with $0.1 \%$ LOD. Since the measurement is made via fiber optic cables, no electrical signals are introduced into the sampling region or needed for the measurement, thus making the sensor free from electrical interferences. Due to the utilization of the complete optical spectrum and chemometric analysis techniques to determine hydrogen concentrations, a more robust sensor is obtained when compared to electrical sensors based on a single voltage or current measurement. Various parameters associated with our existing sensor are being evaluated in order to improve the reliability and robustness of a system deployed under the conditions expected for this application. 


\section{DISCUSSION}

\section{Measurement System}

A schematic representation of the system is shown in figure 1. Light from the tungsten-halogen source is launched down one fiber to the sensor and the reflected light is transported back by the second fiber to the spectrometer where all wavelength components are measured simultaneously. At the sensor head light from the first fiber, which is placed at the focal point of the lens, is collimated by the lens, reflects off of the metal surface and is focused back to the second fiber. This arrangement increases the reproducibility of the measurement over those where the coating is placed directly on the fiber end-face since the increase in surface area of the mirror means any small crack or imperfection has a correspondingly small effect on the reflectivity. The preferred spectrometer used in these studies is a Carl Zeiss model \# MCS500. It is a diode-array based spectrometer with 512 pixels (356nm to $766 \mathrm{~nm}$ ) and has several unique features: pixel resolution is $0.8 \mathrm{~nm}$; the optical resolution is $2.4 \mathrm{~nm}$; and it has a built in fiber optic cross-section converter which reduces wavelength registration error caused by variations in positioning the input fiber. The spectrometer body is constructed of ceramic, which reduces sensitivity to differential thermal expansion caused by ambient temperature changes. This leads to a temperature-dependent spectral drift of less than 0.1 $\mathrm{nm}$ per $10^{\circ} \mathrm{C}$. The analog-digital input electronics yield a wide dynamic range and, coupled with the low noise diode array, allow for very low absorbance measurement noise of less than 0.00005 A. U. RMS.

An experimental system was constructed in order to expose the sensor to mixtures of hydrogen in nitrogen or air with the actual concentration set under computer control. The test cell volume was kept to less than $5 \mathrm{cc}$ and the base flow rate of the nitrogen or air was fixed at $2500 \mathrm{cc} / \mathrm{min}$ as measured by a rotometer in order to minimize the time between a computer change in concentration and the appearance of the change in the cell. The relative changes in the hydrogen concentration were held to a precision better than $0.1 \%$ since the hydrogen flow rate was controlled with a computer interfaced MKS mass flow controller. However, the absolute accuracy in hydrogen concentration was limited by the rotometer setting.

\section{Sensor Coatings}

\section{Sputter System}

A detail of the sensor is shown in figure 2 where it is seen that the alloy layer is deposited directly on the flat surface of a quartz lens. In order to custom produce these coatings we procured and constructed a DC planar magnetron sputtering system. The system was used to fabricate and test various multilayer palladium and palladium alloy thin-film structures for use in the fiber-optic hydrogen sensor. The sputter unit is a dualsource system and allows for rapid, precise deposition of metal and metal alloy layers onto a substrate with 0.1 angstrom precision. The configuration of the sputter system allows for co-deposition of metals in order to generate custom alloy stoichiometries or, alternately, a commercially prepared alloy of the desired stoichiometry can be used as a single target. We are also able to sputter dielectric targets using an rf power source and 
have the capability to create metal oxides by sputtering metal targets in the presence of an oxidizing atmosphere.

\section{Pure palladium and palladium alloy coatings}

Our current sensor is based on coating a collimating lens with a palladium-alloy film with a thickness in the range of 200-1000 angstroms. Pure palladium sensors have been prepared and tested with and without protective polymer coatings. Typical responses of uncoated sensors to hydrogen concentrations in the range of $0.5 \%$ to $3 \%$ hydrogen in nitrogen and air are shown in figures 3 and 4, respectively. Repetitive cycling of a sensor to the same hydrogen concentration yields the same reflectivity with a relative noise equivalent concentration of about $0.1 \%$. The response times needed to reach $90 \%$ maximum depend on the hydrogen concentration and are as fast as 25 seconds for $3 \%$ hydrogen. The recovery time is dependent upon both the hydrogen concentration and the ambient temperature. For instance, recovery times are increased 2-3 times over the $35^{\circ} \mathrm{C}$ response time when the temperature is lowered to $10^{\circ} \mathrm{C}$. It was observed that for some sensors (no polymer coating), an initial negative-going trend in reflectivity preceded the normal response when the sensor had been standing in air for an extended amount of time. It is assumed that this response is due to the fact that uncoated sensors will pick up adsorbed gases when left exposed to ambient air. However, the standard response level is reached in the reflectivity after the initial negative response and, in addition, the effect is not observed in subsequent short duration cycles This initial effect depends upon the alloy and is more pronounced for palladium -silver alloy than for pure palladium.

In order to test the effects of polymer coatings on response times and initial perturbations of the response, a sensor consisting of a metal film sputtered from an alloy target with 95\% Pd and 5\% Ag was prepared and tested. As expected, the initial negative effect can be seen in figure 5 for the uncoated sample after standing in air overnight. Since it is well known that hydrogen will permeate thin films of some organic substances readily while larger molecule gases will not, several sensors were coated with thin films of the organics teflon and paralyene. A spin-coating technique was used to put down thin (1500 angstrom) films of clear teflon by dissolving amorphous teflon in a solvent. The suppression of this negative effect by the teflon coating is clearly illustrated in figure 5. It was determined that the response time was not affected significantly by the coatings and the typical response for a coated palladium sensor in air is shown in figure 6 . It has been shown here that these sensors give a good response time and adequate sensitivity to measure $0.1 \%$ hydrogen.

\section{Yttrium-palladium layered structures}

The desire for even greater sensitivity and robustness has led us to look at other sensor designs. Joint work with the DOE Kansas City Plant has identified a layered sensor structure based on yttrium with a palladium overcoat that appears to meet these requirements and we have been determining the effect of such construction parameters as quality of the sputtering atmosphere and film thickness on its properties. This has led to the evaluation of a yttrium thin film sensor for hydrogen sensing using the apparatus described above. We have acquired the necessary sputtering sources and have developed the capability to prepare these layered optical sensors in our sputtering facility. 
Yttrium/palladium films were deposited onto various lens and planar substrates using a DC planar magnetron sputtering system under high vacuum conditions using argon as a sputtering gas. Films were prepared with thicknesses ranging from 1000 angstrom to 5000 angstrom $Y$ with 200 angstrom Pd overcoat to protect the yttrium, which is readily oxidizable in air. The theory behind the sensor response is this: molecular hydrogen will diffuse through and dissociate in the palladium layer and form various hydrides with the yttrium layer, depending upon the hydrogen partial pressure. The sensors were configured in both reflection mode and transmission mode, and final evaluation was performed in transmission mode for these sensors. Each coated lens was mounted into a swage-lock fitting and connected into a chamber with valves attached to allow pumping out via a rough pump and introduction of dry air or nitrogen. A pressure gauge was affixed to the pump line so that partial pressures could be measured during back-filling of the chamber.

A silica lens with the end coated with 3000 angstrom Y and $200 \mathrm{~A} \mathrm{Pd}$, was fitted into the test chamber and exposed to various concentrations of hydrogen in various mixtures of air and nitrogen.

Standard $0.996 \% \mathrm{H} 2$ in $\mathrm{N} 2$ was used as well as dilutions (1:1) in air and $\mathrm{N} 2$ in order to test the differences in response due to the presence of oxygen, which appears to affect the recovery rate of the sample to its pre-exposure state. It appeared that the response was very reproducible to these small $(<1 \%)$ concentrations of hydrogen, and the time scale of the response was on the order of 5 minutes for each run. Figure 7 represents the single wavelength responses at $484 \mathrm{~nm}$ and $810 \mathrm{~nm}$, which are the corresponding transmission peaks that grow in as the sensor is exposed to hydrogen. Notice the difference between the responses, as shown by the red curve. In order to more fully understand the response variance associated with exposure to hydrogen, a singular value decomposition was performed, which decomposes the response matrix into orthogonal vectors representing individual orthogonal contributions to the overall variance. Figure 8 details the score (variance in the experiment time course space) and loading (wavelength space variance) responses for the series of hydrogen pulses, and it is clear that the there are two mechanisms responsible for the overall response of the film to hydrogen. The lower transmission peak appears to lag behind the peak at $810 \mathrm{~nm}$ and the largest lag is seen during large changes in the hydrogen partial pressure. We have determined that the response times do not change significantly as a function of the $\mathrm{Y}$ film thickness in the range of 1000-5000 angstroms but are comparable to the pure palladium sensors ( 100 seconds to $90 \%$ of maximum). The figure captions give more detailed information about the specific response for each phase of the experiment.

It is postulated here that these effects may be the result of thin film optical interference, or etalon effects, or that the broad minimum in the transmission spectrum, which corresponds to an optical absorption, could be the result of the mixture of yttrium and yttrium oxide, as the sputtering facility did not achieve low enough oxygen partial pressures to sputter pure yttrium. The positions of the transmission peaks depend on hydrogen concentration and work is still in progress to determine what part of these spectra are due to etalon effects and what are due to intrinsic absorption.

These preliminary measurements indicate that these sensors may be useful down to less than $0.05 \%$ hydrogen. Work will continue to characterize and understand these sensors because of their potential for greater sensitivity and robustness, which is caused by greater wavelength dependence of the spectra over those of the palladium alloy films. Our films look identical to those prepared at KCP in that the yttrium film does not look 
metallic regardless of great effort to achieve a low oxygen atmosphere before sputtering in ultra-pure argon. Nevertheless the response to hydrogen is large, as evidenced by the films changing from dark brown to clear upon exposure to hydrogen, evidence that these films exhibit the yttrium hydride optical switching reported in Nature magazine in 1996.

\section{Improvements in multiplexing sensors}

Work on improving the spectroscopy measurement system for the hydrogen sensor has progressed also. A spectrometer based on an array detector for multi-sensor input was evaluated for use as an alternative to using a line-type detector coupled with a physical translation-stage multiplexer. In this latter configuration, which is the normal arrangement, the fiber carrying light from the light source and the fiber carrying the reflected light to the spectrometer are moved into alignment with the fibers carrying light to and from a desired sensor using a computer-controlled, mechanical translation stage. In the new arrangement the fibers carrying light from the separate sensors are all imaged simultaneously onto different regions of the CCD array by packing the fibers into a vertical bundle and imaging them onto the spectrometer slit. Each fiber yields an independent spectra on a corresponding stripe of the detector. This has the advantage over the mechanical multiplexer in that there are no moving parts and a reference spectra can be acquired simultaneously with the sample spectra so that no drift effects are encountered due to any time lag. An adapter to connect seven fibers to a slit shaped bundle has been constructed and tested with good results. The standard chemometric software package was modified to make the correspondence between the multiplexer position and a region on the detector.

\section{CONCLUSION}

It is clear from these results that a fiber optic reflectivity measurement of thin films of pure palladium can be used as a means to measure hydrogen concentrations remotely without introducing any electrical signals or wires into the measuring region. This means the sensor will be immune to electrical noise and further will not interfere with any other electrical sensors. The measurement of the reflectivity at multiple wavelengths enables the identification of a malfunctioning sensor and, in addition, makes it possible to develop robust chemometric algorithms for background corrections and verification of operation. Further, we have seen that the sensor surface may be protected from reactive gases by coating the sensor with a semi-permeable membrane. The actual long term protection afforded by these coatings needs to be investigated further with regard to actual gas and vapor species that are likely to be encountered in use. The sensitivity of the pure palladium sensors, although adequate is not as great as one would desire. Better optical geometries and methods of interaction with the excitation light in order to increase the sensitivity to changes in the palladium reflectivity or index of refraction should be investigated but it also looks promising to continue the investigation of the yttrium palladium layered structures for this purpose. Although the reproducibility of the reflectivity versus concentration calibration is good on the short term, long term studies need to be carried out in order to test the stability and ruggedness of a sensor in a real application environment. 


\section{SUMMARY}

- We have procured and constructed a dual source DC planar magnetron sputtering system.

- Sensors consisting of pure palladium films with a 20 angstrom chromium underlayer have been constructed and the adhesion was observed to be improved.

- A sensor consisting of a film sputtered from an alloy target with $95 \% \mathrm{Pd}$ and $5 \% \mathrm{Ag}$ was prepared and tested.

- Sensors coated with thin films of teflon and paralyene were prepared and tested and it was determined that the response time was not affected significantly by the coatings.

- The PdAg sensor which showed the greatest negative (cleaning) response after setting in air was observed to be protected from this effect in the short term.

- Work on improving the spectroscopy measurement system for the hydrogen sensor has progressed also. A spectrometer based on an array detector for multi-sensor input was evaluated for use as an alternative to using a line-type detector coupled with a physical translation-stage multiplexer

- We have acquired the sputter sources and developed the technique to prepare sensors based on palladium/yttrium layered structures. Sensors with several different thicknesses of the yttrium layer have been prepared and tested.

- Sensors based on this layered structure were shown to give greater sensitivity than the pure palladium film sensors allowing measurements down to $0.05 \% \mathrm{H} 2$ in air

- These layered sensors need the presence of air in order to recover quickly after exposure to hydrogen and this leads to baseline drifts that need to be overcome. 


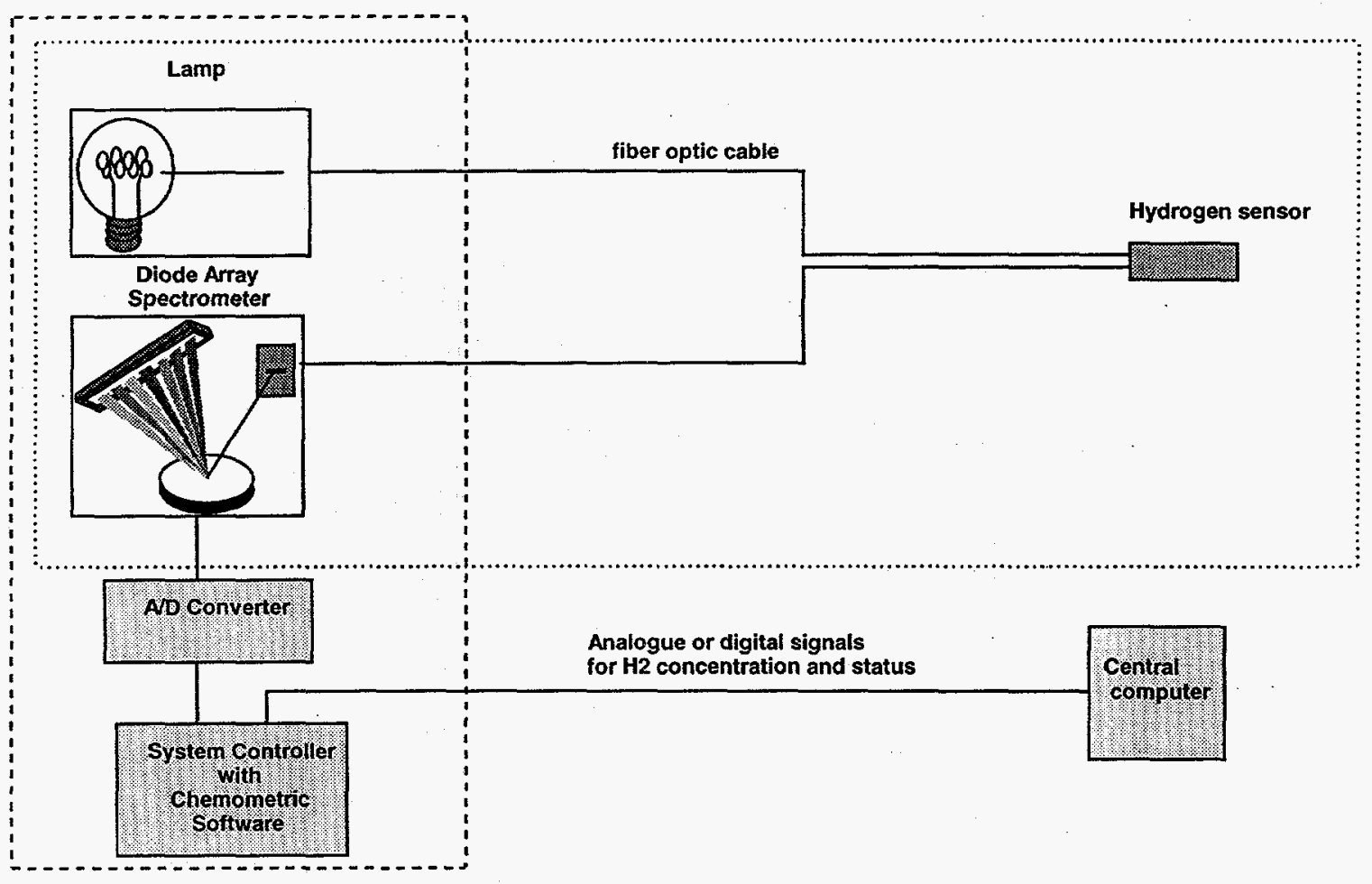

Figure 1. Schematic of the complete fiber optic thin film hydrogen sensor system. 


\section{Fiber-Optic Hydrogen/Temperature Sensor}

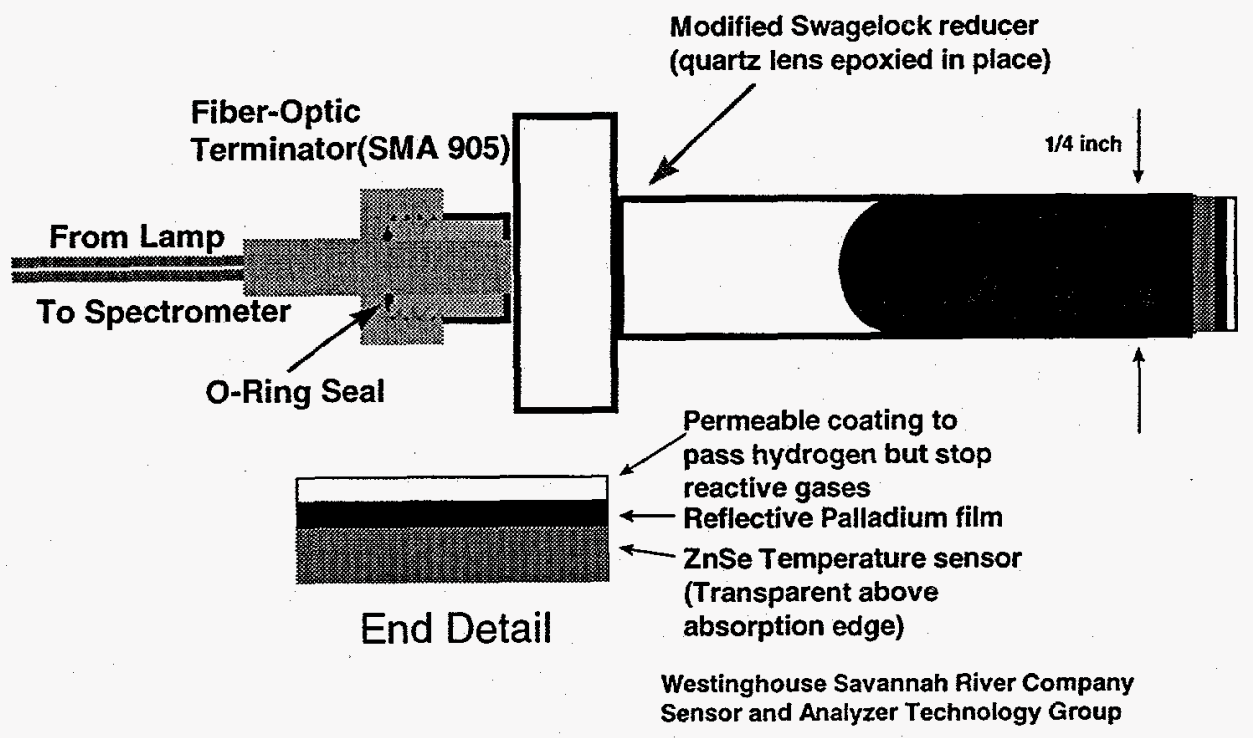

Figure 2. Schematic of the thin film palladium sensor. The inset shows a close-up of the layered coatings on the end of the lens(the integral $\mathrm{ZnSe}$ temperature sensor is proposed but is not included for this work) 


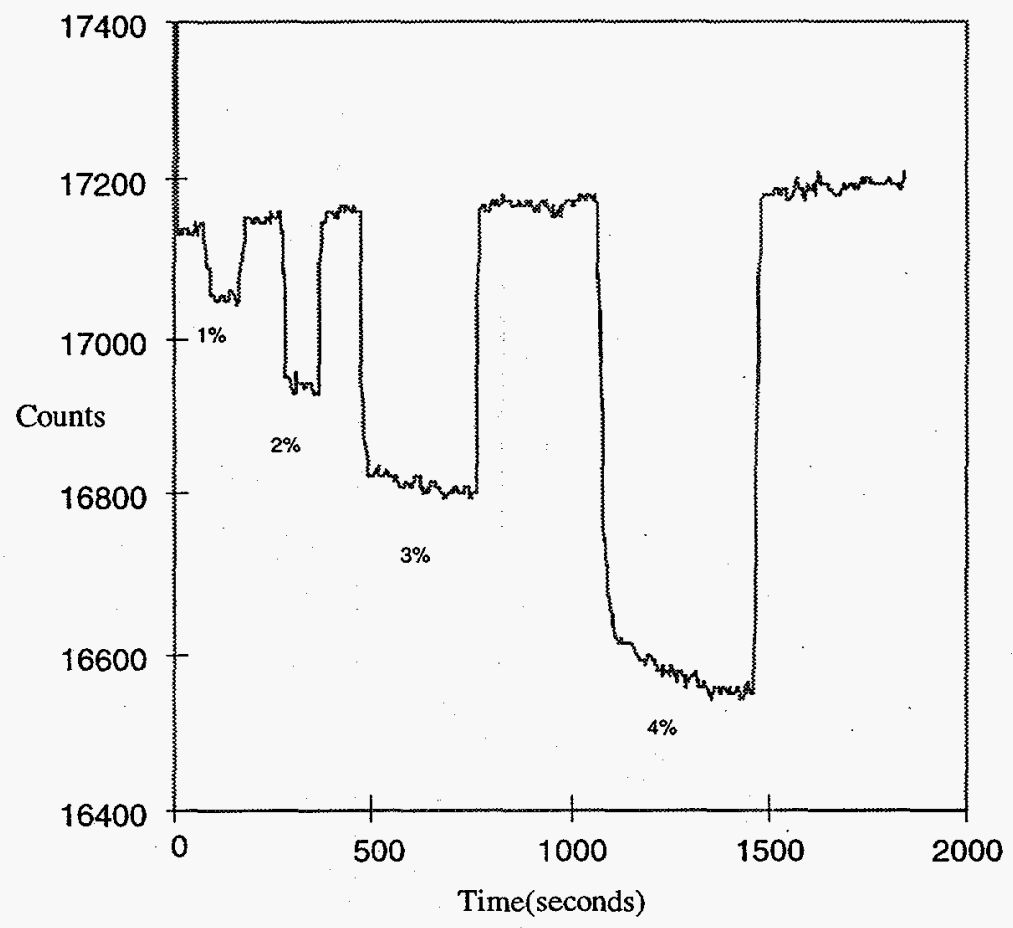

Figure 3. Reflectivity(counts) of a pure palladium sensor at 635 nanometers versus time(seconds) in response to the indicated pulse concentrations of hydrogen in air . 


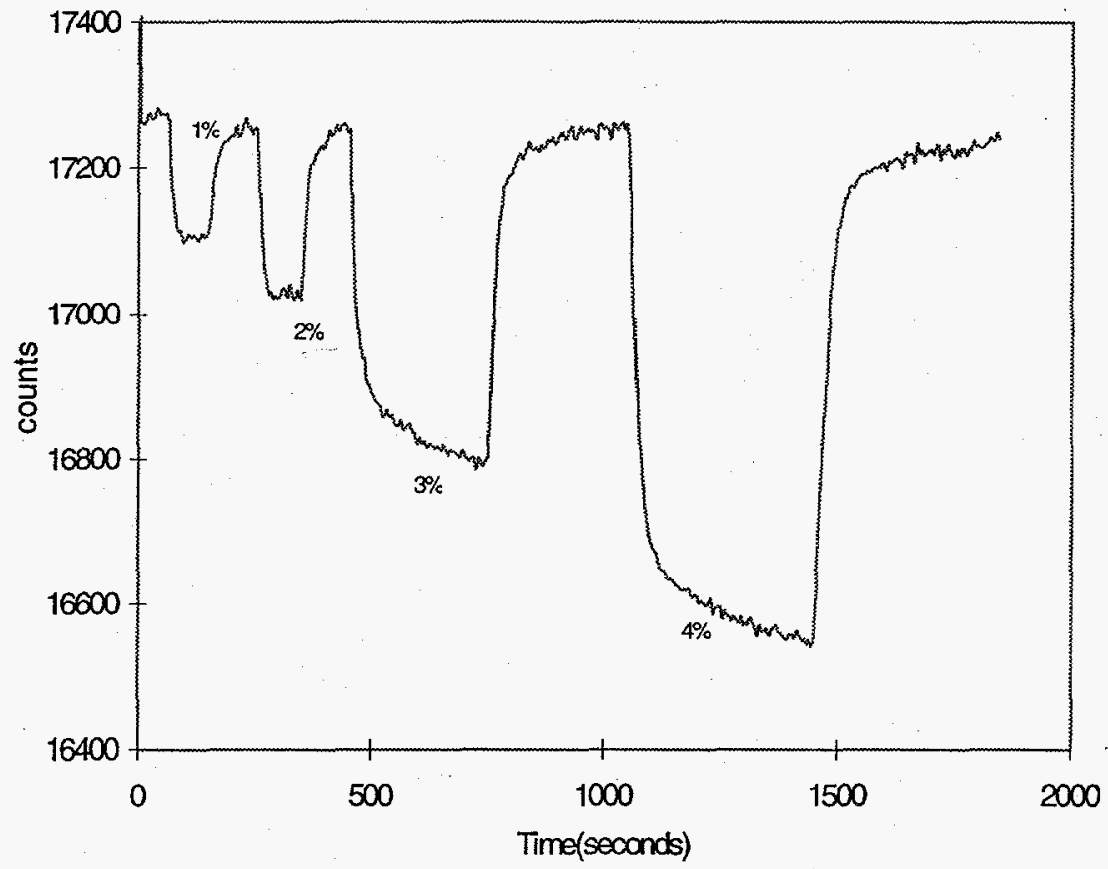

Figure 4. Reflectivity(counts) of a pure palladium sensor at 635 nanometers versus time(seconds) in response to the indicated pulse concentrations of hydrogen in nitrogen 


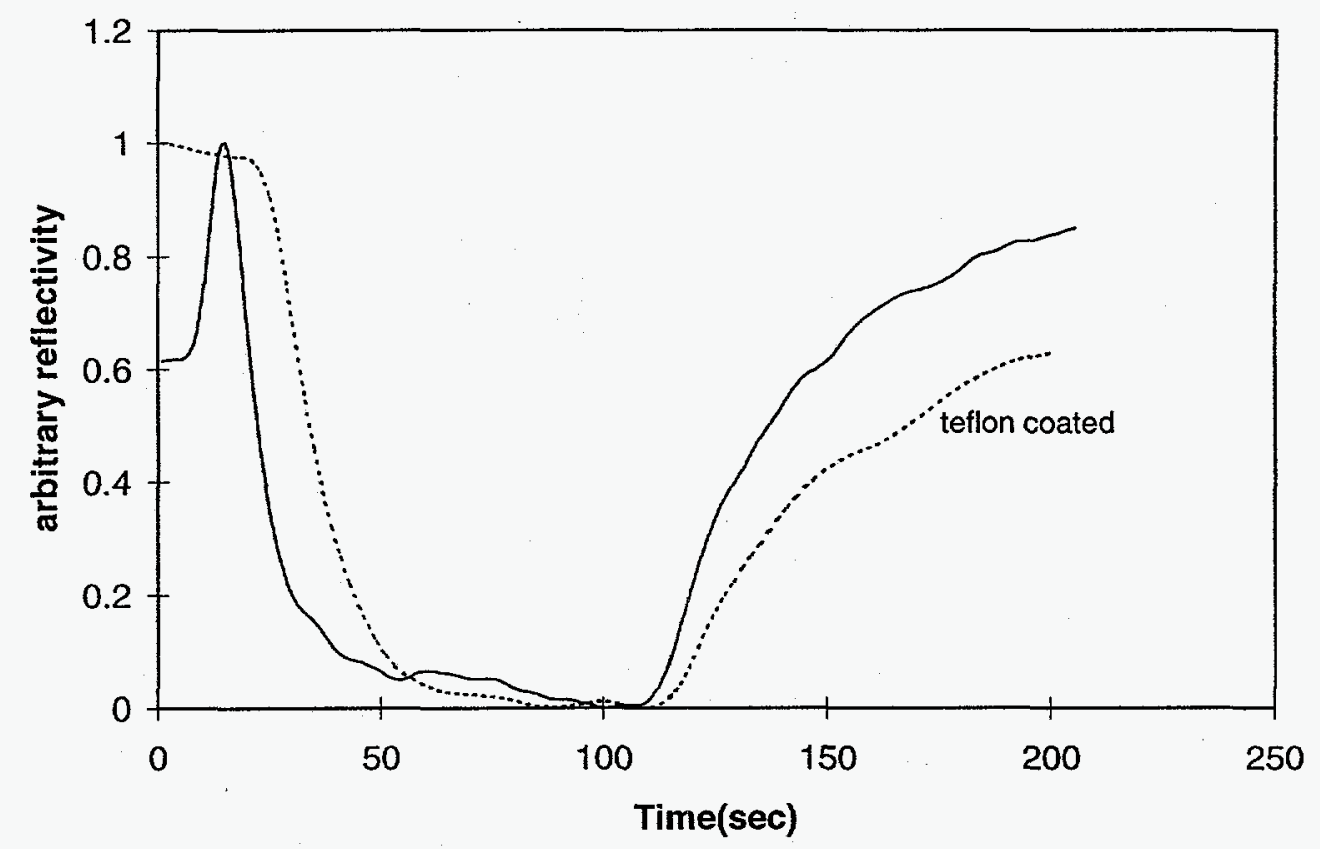

Figure 5. Reflectivity(counts) of a 95\%palladium / 5\% silver sensor at 635 nanometers versus time(seconds) in response to a pulse of $.5 \%$ hydrogen in air after setting in air for an extended period. The curve with the initial increase in reflectivity is the uncoated sensor while the curve for which this spike is suppressed is coated with $\sim 1500$ angstroms of teflon. 


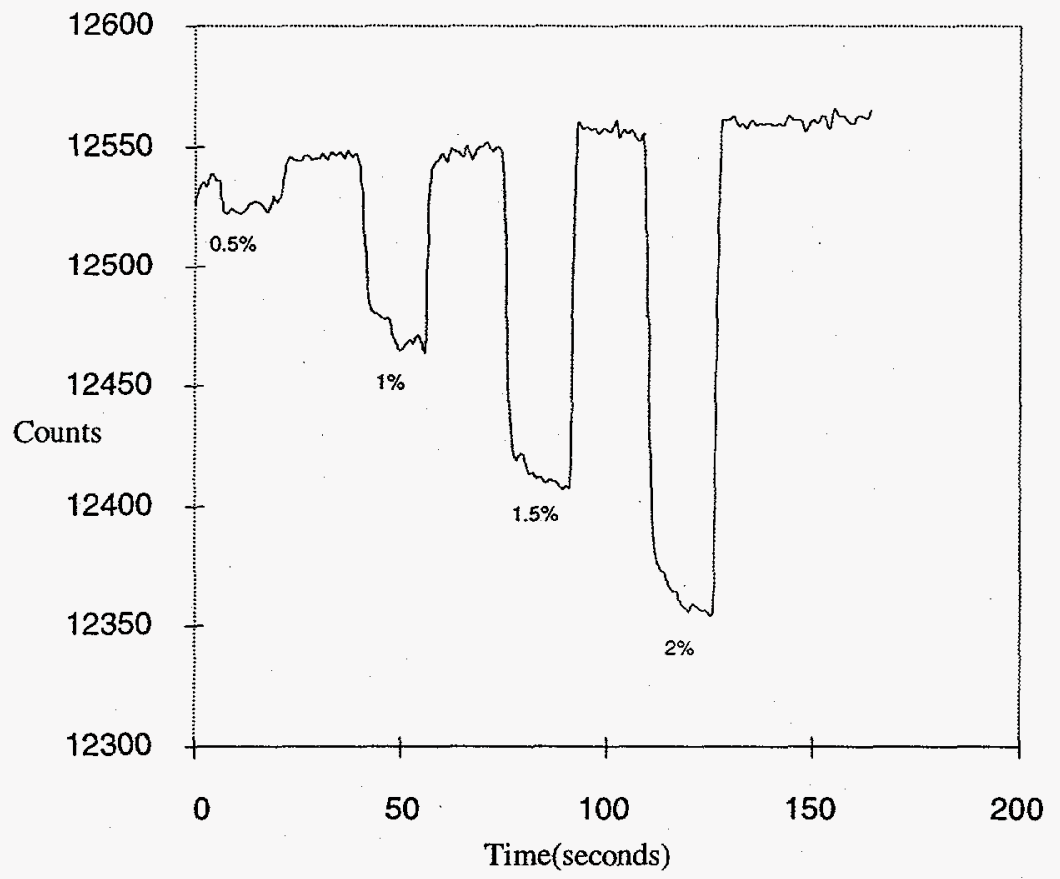

Figure 6. Reflectivity (counts) of a pure palladium sensor at 635 nanometers versus time (seconds) in response to the indicated pulse concentrations of hydrogen in nitrogen. 


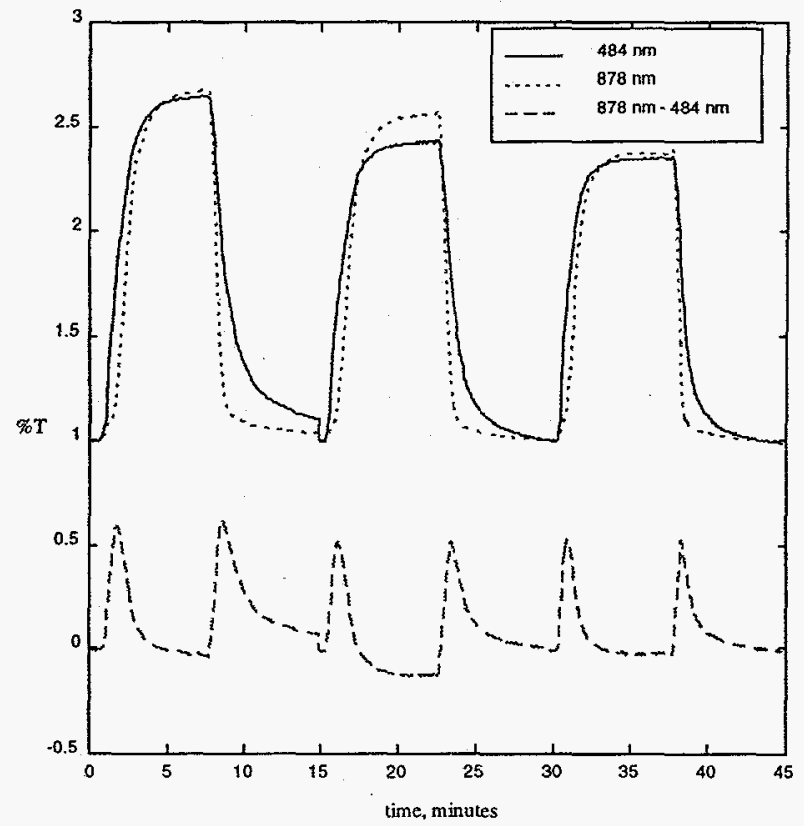

Figure 7. Response of 3000 Angstrom $\mathrm{Y} / 200$ Angstrom Pd film to successive hydrogen pulses. $.996 \% \mathrm{H}_{2}$ in $\mathrm{N}_{2}$ was used as a sample gas for first run, diluted 1:1 in $\mathrm{N}_{2}$ for second run, and diluted 1:1 in air for the final run. Both peaks are tracked as the sample is let into and released from the chamber, and the red curve below is the amplitude difference between the peak maxima. 

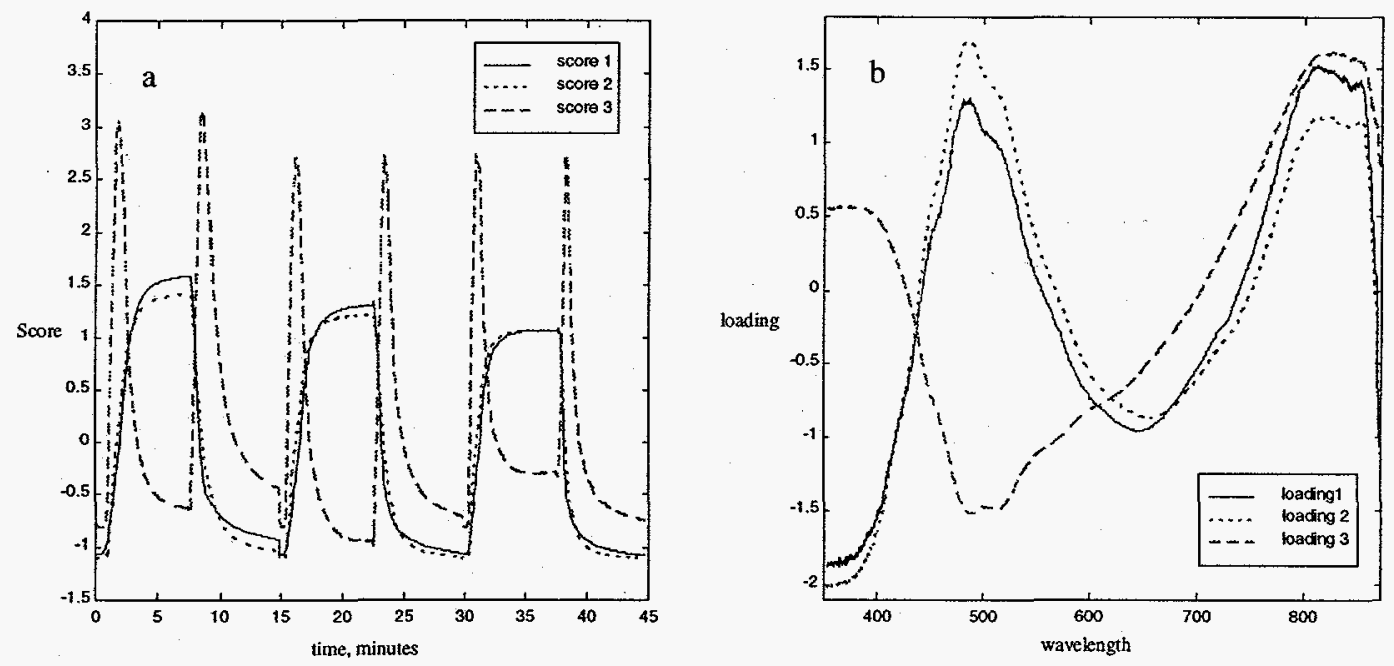

Figure 8. Singular Value Decomposition of the response of the Y/Pd film to sequential hydrogen pulses. (a) Scores one and two look very similar to the single wavelength magnitude trend and score 3 resembles the difference between the peaks' response profiles. These can be correlated to the wavelength response by examining the corresponding loading vectors (b). The transmission peaks are correlated with each other as hydrogen is added and removed from the cell, but to a differing degree, i.e. in score one the peak at 810 has a higher loading, or a greater response, and for score 2 the peak at $484 \mathrm{~nm}$ has a higher loading. This indicates that they respond at different rates to the presence of hydrogen. The intermediate area, where rapid changes in hydrogen partial pressure occur, is described by loading three, where the two transmission peaks are anticorrelated in their response. This is due to the more rapid response of the peak at $810 \mathrm{~nm}$ relative to that of the peak at $484 \mathrm{~nm}$. This clearly indicates that two mechanisms are present in the optical response of the multilayer Y/Pd hydrogen sensor. 